\title{
Numerical modeling and digital image correlation strain measurements of coated metal sheets submitted to large bending deformation
}

\author{
Laurent Duchêne $^{\mathrm{a}}$, Amine Ben Bettaieb $^{\mathrm{b}}$, Victor Tuninetti $^{\mathrm{c}}$ \\ and Anne Marie Habraken ${ }^{d}$ \\ ARGENCO Department, MS2F Division, University of Liège, \\ Chemin des Chevreuils 1, 4000 Liège, Belgium \\ a.duchene@ulg.ac.be, bamine.benbettaieb@ulg.ac.be, 'cv.tuninetti@ulg.ac.be, \\ danne.habraken@ulg.ac.be
}

Keywords: Finite element method, solid-shell elements, sheet forming, coated metallic sheet, digital image correlation.

\begin{abstract}
The recently developed SSH3D solid-shell element [1], which is based on the Enhanced Assumed Strain (EAS) and the Assumed Natural Strain (ANS) techniques, is utilized for the modeling of a severe bending sheet forming process. To improve the element's ability to capture the through thickness gradients, a specific integration scheme was developed. In this paper, the performances of this element for the modeling of the T-bent process were assessed thanks to comparison between experimental and numerical results in terms of the strain field at the outer surface of the sheet. The experimental results were obtained by Digital Image Correlation. It is shown that a qualitative agreement between experimental and numerical results is obtained but some numerical parameters should be optimized to improve the accuracy of the simulation predictions. In this respect, the influence of the penalty coefficient of the contact modeling was analyzed.
\end{abstract}

\section{Introduction}

Nowadays, coatings are increasingly used in the industry in order to enhance some properties of the products or to bring new properties. For instance, steel sheets are coated for corrosion protection, for aesthetic reasons or for specific applications... Additionally, for an increased productivity, pre-coated sheets are more and more exploited. In this case, the coating is applied on the flat sheet and the obtained composite system (sheet - coating) can be given the desired shape by sheet forming processes. The main drawback of this technique is that the coating is also submitted to the deformation linked to the forming process, which can yield some problems such as decohesion or failure of the coating. These limitations in the deformation admissible in the coating before such defaults occur must therefore be studied to enhance the robustness of these new forming techniques. The numerical modeling of forming processes on pre-coated sheets is consequently a research topic which recently received an increasing interest. Indeed, the modeling permits to derive crucial information concerning the ability of the coating to sustain the forming process. For instance, the stress and strain fields can be obtained and, more specifically, their gradients along the thickness of the coating and the stresses at the interface between the sheet and the coating can be directly related to the decohesion phenomenon.

In this research work, the numerical simulation were performed with the recently developed SSH3D solid-shell element implemented in the home-made LAGAMINE finite element code. This element is based on the Enhanced Assumed Strain (EAS) technique and the Assumed Natural Strain (ANS) technique. These techniques permit to avoid locking problems even in very bad conditions (nearly incompressible materials, very thin elements conducting to large aspect ratios, distorted element geometry...). The EAS technique artificially introduces additional degrees of freedom (DOFs) to the element. In the current configuration of the element, up to 30 independent DOFs can be added to the 24 classical displacement DOFs (corresponding to the 3 displacements of the 8 
element nodes). Contrarily to the nodal displacements, these additional DOFs are not linked between adjacent elements, so that they can be eliminated at the element level during the computation of the solution (before the assembling procedure). Nevertheless, they permit to increase the flexibility of the element which is very efficient for several locking issues. On the other hand, the ANS technique modifies the interpolation scheme for particular strain components. This technique is useful when shear and curvature locking problems are encountered. The ANS technique proved to eliminate the transverse shear locking from the element in bending dominated situations. In the current configuration of the element, four different versions of the ANS technique were implemented in the SSH3D element. Besides, a numerical integration scheme dedicated to solid-shell elements was implemented. It uses a user-defined number of integration points along the thickness direction, which permits to increase the element accuracy with a mesh containing a reduced number of elements along the thickness direction.

In this paper, the quality of the element results is assessed through the simulation of a very large strain forming process, i.e. the T-bent. During this process, the coated metal sheet is folded with an angle of $180^{\circ}$, resulting locally in a very large bending deformation. The metal sheet and the coating are modeled (separately) with SSH3D elements, while contact elements permit the modeling of the interaction with the forming tools. The numerical results are then confronted to experimental measurements. Indeed, the strain field at the outer surface of the bent (where the sheet is coated) is measured with a digital image correlation technique, which had to be set up for this particular application where very large strains have to be measured on a narrow surface (where the sheet is folded).

\section{Formulation of the SSH3D solid-shell element}

The Enhanced Assumed Strain (EAS) technique. This technique was first introduced by [2] in order to avoid various types of locking. For instance, it is particularly efficient for volumetric locking and transverse shear locking. The formulation of an element using the EAS technique departs from the three field $\mathrm{Hu}$-Washizu variational principle and the crucial point of the technique is the enlargement of the strain field according to:

$$
\varepsilon=\varepsilon^{c o m}+\varepsilon^{E A S},
$$

where $\varepsilon^{\text {com }}$ is the compatible strain field derived from the element nodal displacements thanks to the classical $B$-matrix:

$$
\varepsilon^{\text {com }}=\Delta^{s} u=B(r, s, t) \cdot U
$$

The enhanced part of the strain field $\varepsilon^{E A S}$ is constructed in a similar way using the $G$-matrix and the additional degrees of freedom $\alpha$ related to the EAS technique:

$$
\varepsilon^{E A S}=G(r, s, t) \cdot \alpha=\frac{j_{0}}{j(r, s, t)} \cdot F_{0}^{-T} \cdot M(r, s, t) \cdot \alpha
$$

In equations (2) and (3), $r, s, t$ represent the intrinsic coordinates of the element, $U$ is a vector containing the nodal displacements (corresponding to 24 degrees of freedom), $F_{0}$ is the jacobian matrix related to the transformation between the spatial coordinates and the intrinsic coordinates (the index 0 meaning that it is computed at the center of the element), $j_{0}$ is the jacobian determinant at the center of the element and $j(r, s, t)$ is the jacobian determinant at point. According to equation (3), the additional strain modes related to the EAS technique are included in the matrix $M$ and they are expressed in the intrinsic reference frame as: 


$$
M(r, s, t)=
$$

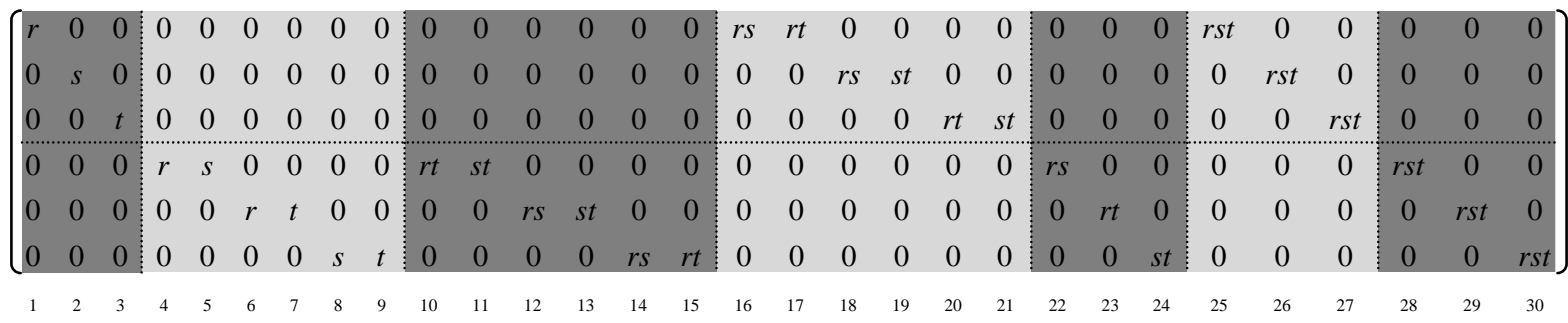

In equation (4), the modes having non-zero terms in the three upper rows are related to the normal strain components and are therefore contributing to the volumetric locking issue. Conversely, the modes with non-zero terms in the three lower rows correspond to shear strains and are used to solve shear locking. As shown in equation (4), the $M$-matrix expands the strain field up to the complete tri-linear field. It results that the EAS element has 30 potential additional modes which can be activated in order to modify the element behavior. Additionally, it is essential that the 30 enhancing strain modes included in equation (4) are linearly independent from the 24 classical strain modes obtained from the compatible strain part (equation (2)). The EAS method has been widely adapted for 2D, 3D and solid-shell formulations [3].

The Assumed Natural Strain (ANS) technique. This technique permits to avoid several locking issues by modifying the interpolation of the strain components in the element. Classically, the strain is computed at the integration point or at any location inside the finite element from the nodal displacements with the $B$-matrix according to Equation (2). For some particular cases, this usual technique yields to inadequate strain values for some components depending on the location where the strain is computed. The ANS technique, originally proposed by [4] for shell elements, suggests to achieve the interpolation of the problematic strain components in two steps. First, these strain components are evaluated by the classical interpolation method at the so-called 'sampling points', where the erroneous values are not likely to be encountered. In a second step, these strain components are interpolated linearly from the sampling points to the integration points (or any location in the element).

In order to assess the efficiency of the ANS technique on the mechanical behavior of the SSH3D element, four different versions of the ANS interpolation have been implemented to date. These versions differ from each other by the number and the locations of their sampling points. Further details can be found in [1].

The integration scheme. An important characteristic of a finite element is the numerical integration scheme. The number and the location of the Gauss points inside the element can have a significant influence on its mechanical behavior. For instance, the reduced integration or the selective reduced integration schemes are often used to avoid volumetric locking issues for hexahedral elements with an isochoric or nearly isochoric material behavior.

In the development of a solid-shell element dedicated to the modeling of thin-walled structures, an improved integration scheme with a large number of integration points along the thickness direction was considered. It is indeed expected that a high gradient of stress and strain along the thickness direction is present during the deformation of thin materials (during e.g. a bending deformation mode). The classical full integration of brick elements (with two integration points along each direction) is not able to accurately capture such large gradients. In this respect, in the SSH3D element, the stress is computed along a user-defined number (ranging from 2 to 10) of integration points along the thickness direction (corresponding to the intrinsic coordinate $t$ ).

\section{Experimental T-bent process}

The T-bent process is a severe bending process in which the sheet is folded at around $180^{\circ}$. Experimentally, the process is achieved in two steps with the device shown in Fig. 1. In the first step, the sheet is bent at around $45^{\circ}$ (depending on the sheet thickness and the operator) as shown in 
the figure. Then, in the second step, the sheet is held manually in order to be pressed between the forming tools until the sheet is folded against itself.

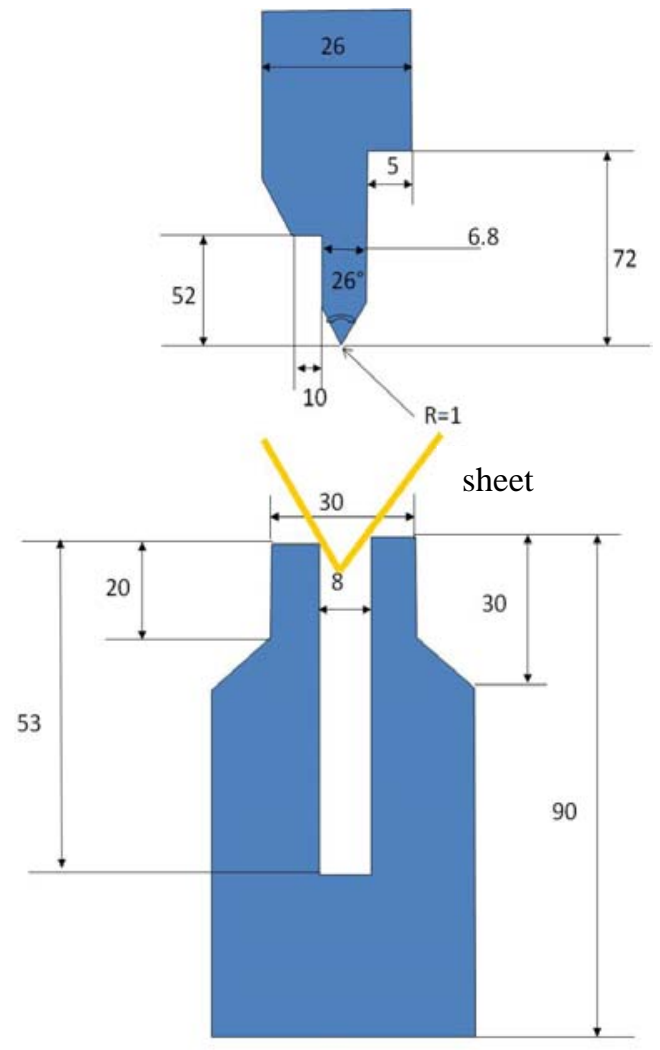

Figure 1: Experimental device for the T-bent (dimensions in mm).

The process can be repeated several times to increase the number of metal layers that are bent against each other. In this paper, we will focus on the first folding called 0T and represented in Fig. 2 (a) and in the second bending called 1T (see Fig. 2 (b)).

(a)

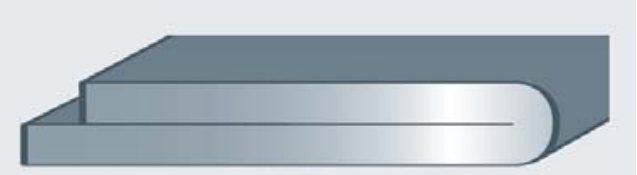

Figure 2: T-bent with one folding 0T (a) and with two folding 1T (b).

The T-bent is used to characterize the capability of the coating to sustain such a large deformation of the metal sheet without failure or decohesion. The coating is thus located at the outer surface of the sheet during the bending. And the strain field in the coating is the keyparameter of the process. In this respect, the Digital Image Correlation (DIC) technique was used to measure the strain field during the T-bent process (at the end of the two steps of the T-bent). As the surface where the measurement is performed is very narrow, a special method was used for the DIC. In order to obtain the desired resolution for the measurement, a predefined pattern was printed on the sheet with an inkjet printer. The pattern, which corresponds to a black and white bitmap file, had to be optimized in terms of dot size and pattern size. It is essential that the different parts of the pattern do not correspond to similar dot sequences, so that the different measurement zones are clearly identified and distinguished from each other. 


\section{T-bent FEM modeling}

For the modeling of the T-bent process, the SSH3D solid-shell element implemented in the home-made LAGAMINE finite element code was used. In the simulations, 6 EAS modes, the third version of the ANS and up to 4 integration points along the thickness direction were used.

Mesh. The finite element mesh consisted of four layers of elements for the steel sheet as shown in Fig. 3. The coating was modeled with two layers of elements (not visible on the figure because of the very small thickness of the coating). Thanks to the symmetry of the process (the geometry of the sheet, the material properties and the loading), only a quarter of the sheet was meshed and adequate boundary conditions were imposed. For the zone where the bending occurs, a refined mesh was used to obtain accurate results.

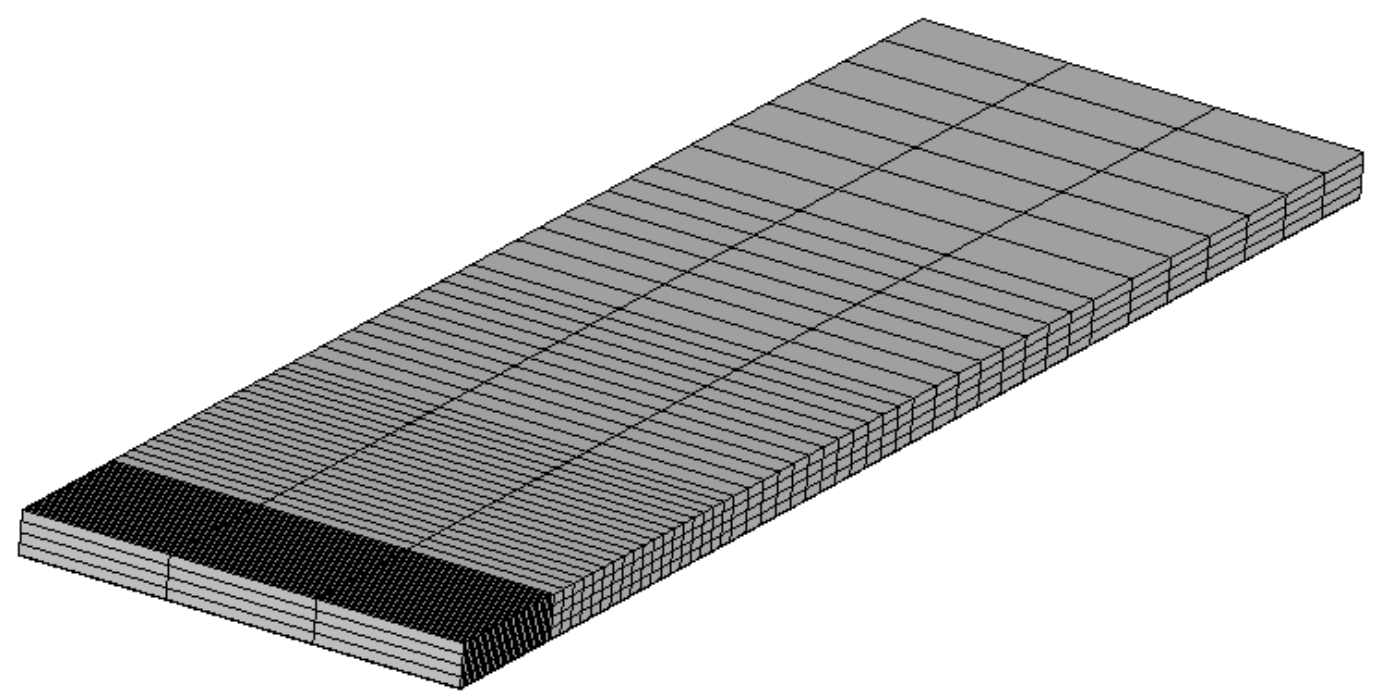

Figure 3: Finite element mesh for the T-bent modeling.

The deformed meshes at the end of the first step and at the end of the T-bent process are shown in Fig. 4.

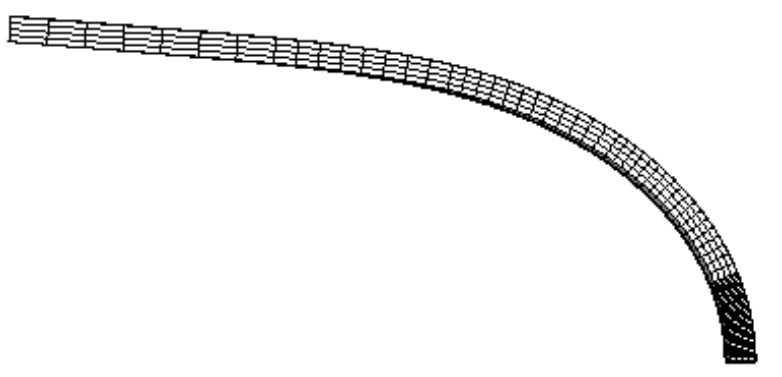

(a) (b)

Figure 4: Finite element mesh at the end of the first (a) and second (b) steps of the T-bent process.

\section{Results and discussions}

In this section, the numerical predictions obtained during the T-bent process simulations are compared to the corresponding experimental results. Fig. 5 shows the strain field on the outer surface of the T-bent process for the 0T case. The maximum principal strain is plotted in the figure, corresponding to the tensile strain related to the bending. A qualitative agreement between experimental and numerical results is obtained. The maximum strain value is located at the symmetry plane of the bending and lower values are gradually obtained when going farther from there. However, the strain measured experimental reaches a value of around 0.628, while the simulation predicts a value lower than 0.37 at the same location on the sheet. This large difference 
can be partly explained by the different behavior of the steel sheet at the vicinity of the folding. As explained later, the penalty method used to model the contact between the sheet and the tools can also induce some inaccuracies in the numerical predictions.

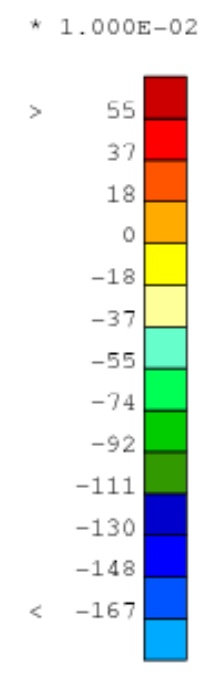

Figure 5: Strain field on the outer surface of the T-bent (0T case) predicted numerically (a) and obtained from DIC experimental measurement (b).

The strain fields for the $1 \mathrm{~T}$ case are shown in Fig. 6. As the bending radius is increased due to the additional sheet inside the bent, the overall strain values are lower than for the 0T case. But the discrepancy between experimental and numerical results also exists for similar reasons.
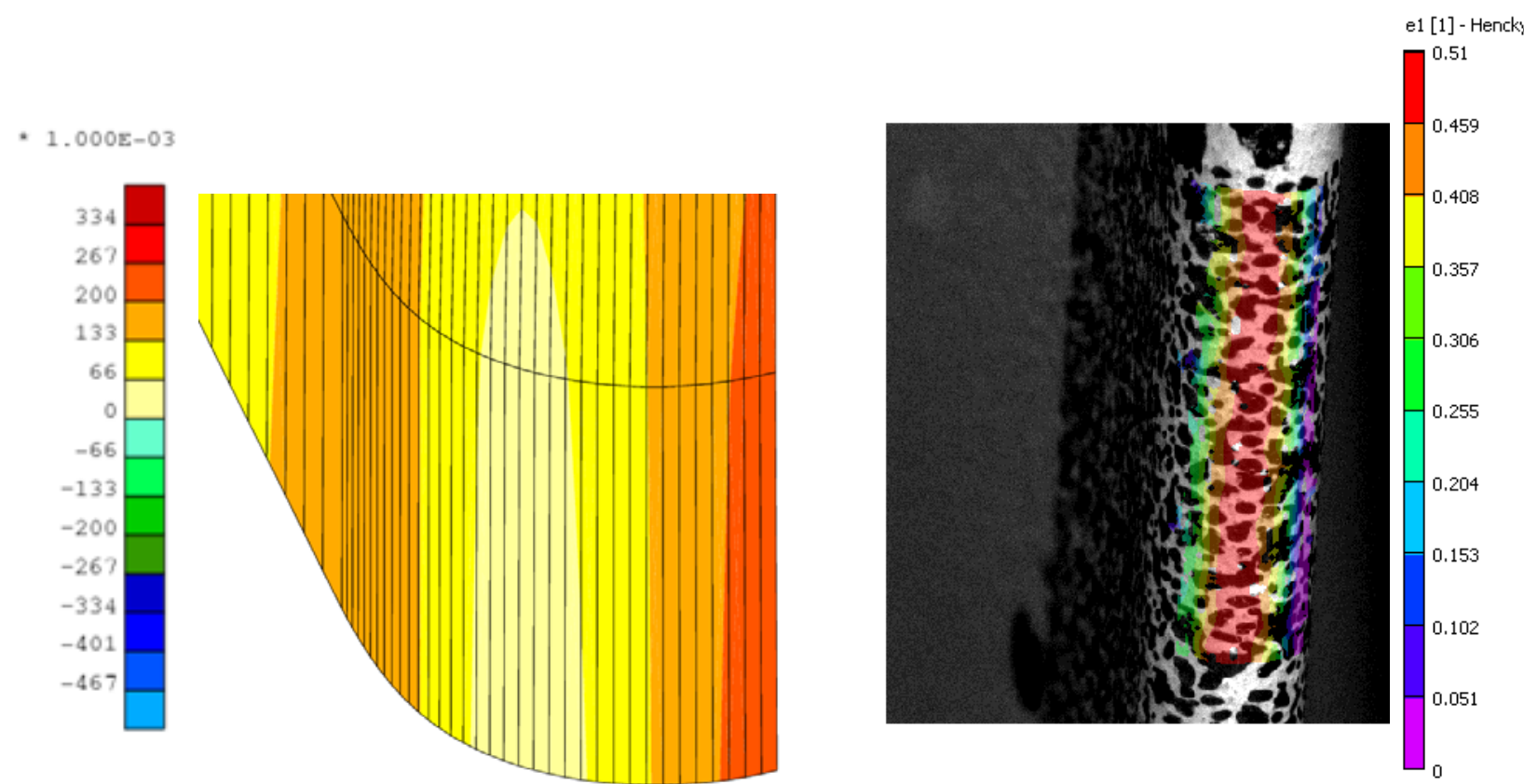

Figure 6: Strain field on the outer surface of the T-bent (1T case) predicted numerically (a) and obtained from DIC experimental measurement (b).

In order to identify the key-parameters affecting the numerical predictions during the T-bent simulations, the influence of some parameters should be investigated. For instance, the influence of the penalty coefficient used for the modeling of the contact between the sheet and the forming tools is presented in Fig. 7. As expected, when the penalty coefficient is increased, the bending condition is enforced in a more pronounced way, especially in the curved part of the bent. 


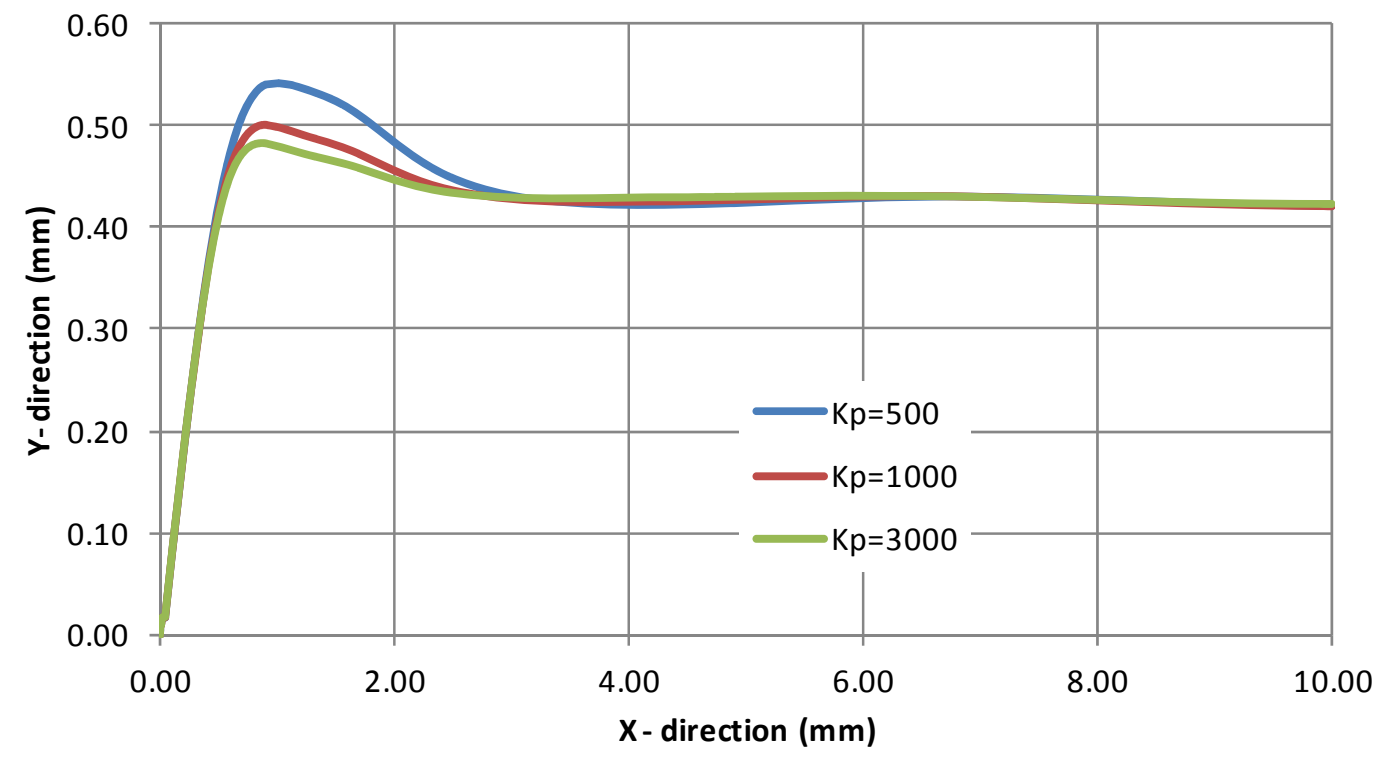

Figure 7: Influence on the geometry of the deformed sheet of the penalty coefficient for the contact modeling during the T-bent simulation (the curves correspond to the outer surface of the sheet).

\section{Conclusions and future works}

This paper presents up-to-date numerical modeling of the T-bent process using the recently developed SSH3D solid-shell finite element. This element is particularly adapted to the modeling of the thin coating layer located at the outer surface of the bending process. It is able to model accurately the stress and strain fields, especially the through thickness gradients and the stress at the interface between the different layers thanks to the large number of integration points along the thickness direction and the EAS technique which is able to capture complex deformation modes.

This research work permitted to validate the SSH3D element for the case of a severe bending process thanks to comparison between experimental measurements of the strain field at the outer surface of the sheet with DIC technique and numerical predictions. The results presented in this paper show that the SSH3D element can be used for sheet metal forming processes modeling. Additionally, pre-coated sheets were investigated in this study.

However, it appears that some improvements of the numerical results could be obtained, especially for the determination of the tensile strain field during bending. These improvements rely on some adjustments of numerical parameters as was shown for the penalty coefficient. Other parameters such as the hardening behavior of the steel or the friction coefficient between the tools and the sheet will be analyzed in further works.

\section{Acknowledgements}

The authors acknowledge the Interuniversity Attraction Poles (IAP) Program P7/21 (Belgian Science Policy). A.M. Habraken also thanks the Belgian Fund for Scientific Research F.R.S.-FNRS for its financial support.

\section{References}

[1] L. Duchêne, A. Ben Bettaieb and A. M. Habraken, Assessment of the Enhanced Assumed Strain (EAS) and the Assumed Natural Strain (ANS) techniques in the mechanical behavior of the SSH3D solid-shell element, in the Proceedings of the international conference COMPLAS XI, 2011.

[2] J.C. Simo, and M.S. Rifai: Int. J. Num. Meth. Engng. Vol. 29 (1990), pp. 1595-1638 
[3] R.J.A. de Sousa, J.W. Yoon, R.P.R. Cardoso, R.A. Fontes Valente, J.J. Gracio: International Journal of Plasticity Vol 23 (2007) pp. 490-515

[4] E.N. Dvorkin and K.J. Bathe: Engineering computations Vol. 1 (1984) pp. 77-88 